\title{
Organismos filantrópicos y no gubernamentales en el desarrollo de nuevas ciencias y tecnologías: el caso de las micro y nanotecnologías en México ${ }^{37}$
}

Philanthropic organizations and non-governmental in the development of new science and technology: the case of micro and nanotechnology in Mexico

\author{
Eduardo Robles Belmont ${ }^{38}$ \\ Dominique Vinck
}

Artigo recebido em para publicação em out/2012 e aceito para publicação em nov/2012.

\section{Resumen}

La emergencia de nuevas ciencias y tecnologías vienen acompañadas de nuevas dinámicas en la producción, el uso y la diseminación de nuevos conocimientos científicos y tecnológicos. Estas nuevas dinámicas se reflejan en la reorganización de las actividades científicas, en la creación o la reorientación de nuevas temáticas en la investigación, en la emergencia de nuevas preocupaciones y debates sobre los riesgos e implicaciones sociales, y en la participación de nuevos actores en el desarrollo de la ciencia y la tecnología, entre otros factores. En este artículo se presentan los resultados del estudio sobre las funciones de un organismo con orígenes filantrópicos y no gubernamental que ha sido central en el desarrollo de los sistemas microelectromecánicos (MEMS) en México. Las funciones que se analizan han sido y son desarrolladas en las diferentes etapas del desarrollo de esta tecnología emergente en este país. Además de mostrar las funciones desempeñadas por este tipo de organismo, este texto se cuestiona sobre la modelización de las relaciones entre las diferentes entidades presentes en el desarrollo e instalación de nuevas tecnologías. En las conclusiones intentamos avanzar algunos elementos para tomar en cuenta estos organismos y enriquecer los estudios sociales sobre las nuevas ciencias y tecnologías.

\footnotetext{
${ }^{37}$ Artigo originalmente apresentado na IX Jornadas Latinoamericanas de Estudios Sociales de la Ciencia y la Tecnología, México, 2012.

${ }^{38}$ Eduardo Robles Belmont, CINVESTAV, Postgrado Transdisciplinario DCTS, 07360 Ciudad de México, México. / Miembro de la Red Latinoamericana de Nanotecnología y Sociedad, ReLANS, México. roblesbelmont@yahoo.fr
}

Dominique Vinck, Université de Lausanne, LABSO - bât Anthropole, Faculté de Sciences Sociales et Politiques, Institut des Sciences Sociales, $\mathrm{CH}-1015$ Lausanne, Suiza. Dominique.Vinck@unil.ch 
Palabras clave: organismos filantropía. Modelos de desarrollo científico y tecnológico. Micro y nanotecnologías. México.

\begin{abstract}
The emergence of new sciences and technologies come with new dynamics in the production, use and dissemination of new scientific and technological knowledge. These new dynamics are reflected in the reorganization of scientific activities, in creating or redirecting new topics in research, in the emergence of new concerns and debates about the risks and social implications, and the participation of new actors in the development of science and technology, among other factors. This article presents the results of the study on the functions of an organism with philanthropic and non-governmental sources that has been central to the development of microelectromechanical systems (MEMS) in Mexico. The functions have been discussed and are developed at different stages of development of this emerging technology in this country. In addition to showing the functions of such a body, this text is questioned on modeling the relationships between the various entities in the development and deployment of new technologies. In the conclusions we try moving some elements to take into account these organisms and enrich social studies on the new sciences and technologies.
\end{abstract}

Keywords: philanthropy organizations. Models of scientific and technological development. Micro and nanotechnologies. Mexico.

\title{
Introduccíon
}

La emergencia de nuevas ciencias y tecnologías vienen acompañadas de nuevas dinámicas en la producción, el uso y la diseminación de nuevos conocimientos científicos y tecnológicos. Estas nuevas dinámicas se reflejan en la reorganización de las actividades científicas, en la creación o la reorientación de nuevas temáticas en la investigación, en la emergencia de nuevas preocupaciones y debates sobre los riesgos e implicaciones sociales, así como en la participación de nuevos organismos en el desarrollo de la ciencia y la tecnología, entre otros factores.

El surgimiento del desarrollo de las micro y nanotecnologías (MNT), que se acentuó a inicios de este siglo, vino acompañado de una serie de potencialidades técnicas y económicas. Estas potencialidades han llamado no sólo la atención de agentes provenientes de los sectores académicos, gubernamentales e industriales. A lo largo de una investigación sobre la emergencia de las MNT en México, hemos constatado la presencia de un organismo con orígenes de carácter "filantrópico". Se trata de la Fundación México - Estado Unidos por la Ciencia (FUMEC), organismo no gubernamental que ha desempeñado funciones importantes en la emergencia y el desarrollo de estas ciencias y tecnologías en este país. 
La presencia de este organismo en el desarrollo de las MNT en México llama nuestra atención por tratarse de un tipo de organismo que no es considerado en los modelos teóricos sobre el desarrollo de nuevas ciencias y tecnologías. En las ciencias sociales diversos trabajos han propuesto modelos sobre las relaciones entre los organismos presentes en el desarrollo de nuevos conocimientos científicos y tecnológicos para estudiar y comprender los cambios tecnológicos. Los modelos más recurrentes en estos estudios son el de la triple Hélice (Etzkowitz y Leydesdorff, 2000), el de los Sistemas de Innovación (Lundvall, 1992), el Modo 2 (Gibbons et al., 1994) y en menor medida en América Latina el Triángulo de Sabato (Sabato y Botana, 1971). Un punto en común de estos modelos es que se centran sobre las relaciones entre agentes provenientes de tres sectores: la universidad, el gobierno y la industria.

La pregunta central en este artículo es sobre cómo modelizar las relaciones entre los diferentes organismos presentes en la producción, el uso y la diseminación de nuevos conocimientos. La hipótesis es que en las nuevas dinámicas de desarrollo tecnológico, organismos diferentes a los tradicionales están cumpliendo funciones en estos procesos. Lo que resulta una articulación diferente entre los organismos presentes en los sistemas científicos y tecnológicos, donde cada organismo cumple una o varias funciones y la articulación del conjunto asegura el funcionamiento del sistema.

Para abordar este estudio, a partir de un enfoque funcionalista, esta investigación caracteriza y analiza las funciones desempeñadas por la FUMEC en el desarrollo de las MNT en México, en diferentes dimensiones: la instalación de infraestructuras científicas, la creación de redes científicas y tecnológicas, la formación de recursos humanos, y la diseminación, la transferencia y la comercialización de nuevos conocimientos. Esta investigación se basa en una serie de entrevistas a científicos, a agentes de la FUMEC y del gobierno, así como en el análisis de reportes y otros documentos relacionados con los proyectos para el desarrollo de estas ciencias y tecnologías.

Los resultados expuestos en este artículo exponen las diferentes funciones desempeñadas por la FUMEC en diferentes etapas y niveles durante el desarrollo de las MNT en México. Esto muestra que existen otros organismos a tomar en cuenta en los estudios sobre el desarrollo de nuevas tecnologías. También, se hace énfasis en la influencia que los organismos filantrópicos y no gubernamentales pueden tener sobre los sistemas científicos y tecnológicos, así como en las políticas públicas que los rigen.

\section{Los organismos filantrópicos y no gubernamentales en el desarrollo científico y tecnológico}

En la literatura académica, principalmente en el campo de la historia de la ciencia, diversos autores se han enfocado en estudiar a los organismos filantrópicos en el desarrollo de diferentes campos de la ciencia y la tecnología. Dentro de esta literatura, hemos identificado dos libros que han 
sido dedicados al estudio de estos organismos en la ciencia y la tecnología (Arnove, 1980; Cueto, 1994). Por otra parte, una serie de artículos sobre estudios sociales de la ciencia y la tecnologías han sido igualmente publicados sobre estudios de caso en América Latina (Vessuri, 1994; Cueto, 1997; Harwood, 2009; Fitzgerald, 1994, Faria y da Costa, 2006; Solórzano, 1994), en Africa (Shrum, 2000; Toenniessen et al., 2008), en Asia (Brown, 1980) y en Europa (Stapleton, 2003; Buxton, 2003: Guilhot, 2007). Dentro de esta literatura, es señalado el lugar que ocupan los organismos filantrópicos en el desarrollo científico y tecnológico como entidades importantes en los periodos de la Primera y Segunda Guerras Mundiales, así como en el periodo de la Guerra Fría (Abir-Am, 2002).

Las acciones de las organizaciones filantrópicas se desarrollan a diferentes niveles y dimensiones. De hecho, los estudios sobre la comprensión de la importancia histórica de estos organismos en la ciencia y la tecnología han revelado que sus principales funciones conciernen el financiamiento de actividades de investigación, así como el financiamiento de nuevas infraestructuras y de la formación de recursos humanos. En algunos casos su presencia se da desde el origen de nuevos campos, siendo uno de los casos más remarcables el desarrollo de la "revolución verde" y su emergencia en los países no hegemónicos (Losego y Arvanitis, 2008), donde la Fundación Rockfeller ha sido un organismo importante en la instalación de estas tecnologías. Otro caso interesante en el cual ha estado involucrada esta misma fundación es la emergencia y el desarrollo de la biología molecular (Abir-Am, 2002). En algunos de los estudios que hemos evocado, los campos donde intervienen las fundaciones filantrópicas se caracterizan por ser áreas factibles y donde los conocimientos producidos presentan un alto grado de aplicación. Además, los cambios políticos y sociales en los contextos de acción constituyen un elemento que también toman en cuenta estos organismos durante las definiciones y ajustes de estrategias para el apoyo orientado a desarrollar nuevas ciencias y tecnologías (Arnove y Pinede, 2007; Faria y da Costa, 2006). Otros dos puntos interesantes ya señalados son sobre la instalación de nuevos modelos institucionales en la ciencia (Fitzgerald, 1994) y sobre la construcción y mantenimiento de la hegemonía sobre los países menos desarrollados (Parmar, 2002a y 2002b; Cueto 1994).

Por otra parte, el libro coordinado por Cueto (1994) se centra sobre la implicación de las fundaciones filantrópicas en el desarrollo científico en América Latina. Una de la hipótesis de esta obra es que la ciencia promovida por estos organismos en América Latina se ha basado sobre "una visión elitista de la historia de la ciencia y de la sociedad estadounidense". Cueto argumenta que los agentes de las fundaciones filantrópicas estadounidenses parten de esta visión elitista en la definición de sus programas de acción. Mas adelante regresaremos en la discusión sobre esta visión de los organismos filantrópicos sobre la ciencia y su influencia en los sistemas políticos en ciencia y tecnología.

Actualmente los campos donde están presentes los organismos filantrópicos son en prácticamente todos los sectores y estos organismos 
como ya se menciono no son nuevos y tienen presencia internacional. Las fundaciones y otros organismos no gubernamentales llevan a cabo actividades en los campos de la agricultura, de la salud, de la biología y hasta de las ciencias sociales, como las ciencias políticas y la economía, por ejemplo. Desde los inicios de la filantropía como la concebimos actualmente, en la década de 1920, han sobresalido las fundaciones Rockefeller, Carnegie y Ford, y últimamente en la escena internacional la Fundación Bill y Melinda Gates, sólo por mencionar algunas.

Los estudios que hemos mencionado muestran la importancia de los organismos filantrópicos en la ciencia y avanzan igualmente diversos elementos importantes que nos permiten mejor comprender las dinámicas de la ciencia y de la tecnología donde tienen lugar las acciones de estos organismos. Antes de pasar con el estudio de caso de nuestra investigación, es pertinente revisar las nociones de organismo filantrópico y de organismo no gubernamental. Esto ya que existe una ambigüedad en ambos, lo que dificulta la calificación de las acciones de éstos y creemos por lo tanto que es importante revisarlos en nuestro estudio.

Hasta ahora hemos utilizado los términos "fundación filantrópica", "organismo filantrópico" y "organismo no gubernamental" para referirnos a las entidades sociales que analizamos en este trabajo que no pertenecen a las esferas institucionales de la industria, de la academia y del gobierno. Aquí es importante señalar que usamos todos estos términos estando conscientes de que existe una ambigüedad en sus definiciones y por este motivo aclaramos el uso que hacemos de éstos. Primero, el término "fundación" es comúnmente usado para hacer referencia a las fundaciones filantrópicas, que son organismos sin ánimo de lucro e independientes en lo económico, lo político y lo organizacional de otras esferas institucionales. Por otra parte, líneas más arriba, también evocamos la presencia de "organismos no gubernamentales" en el desarrollo científico. Sobre este segundo término en la literatura académica es común clasificar a las fundaciones filantrópicas como "organismos no gubernamentales". Sin embargo, no todos los organismos llamados "fundación" son "no gubernamentales", ya que hay en efecto organismos gubernamentales que llevan este término en su nombre. Por ejemplo, la Nacional Science Fondation en Estado Unidos, que es la agencia estatal en este país que es responsable de "promover el progreso de la ciencia; hacer avanzar la salud nacional, la prosperidad y el bienestar". Finalmente, entendemos como "fundaciones filantrópicas" a los organismos que llevan a cabo, en diferentes sectores, iniciativas y acciones para el desarrollo a fin de mejorar las condiciones de vida del hombre.

Las definiciones de los términos que venimos de mencionar han sido la base para que califiquemos a la FUMEC, organismo que estudiamos en este trabajo, como un organismo con orígenes filantrópicos. En el siguiente punto expondremos los orígenes y el perfil actual de la FUMEC, donde intentaremos mostrar la evolución de este organismo que actualmente juega un papel importante en el desarrollo de diversos sectores en la ciencia y la tecnología en México. 


\section{La Fundación México - Estado Unidos por la Ciencia}

\section{Origenes filantrópicos y su evolución}

La Fundación México - Estados Unidos por la Ciencia (FUMEC) fue creada en 1992 como un organismo no gubernamental. La iniciativa para la creación de la fundación ha sido atribuida al Congresista estadounidense George E. Browm Jr., quien había hecho la proposición de crear una fundación que abarcara toda América Latina. Esto implicaba el compromiso de varios países, sin embargo las condiciones para una iniciativa de esta envergadura no eran favorables y el solo país con quien esta iniciativa fue posible fue México.

La FUMEC la calificamos como una fundación filantrópica ya que los motivos de su creación son característicos de la filantropía. Esto se refleja en el "principio rector" de la fundación expresado en el segundo reporte de actividades de la FUMEC por su Presidenta: "un espíritu de cooperación y de participación en ciencia y tecnología puede, y logrará, ayudar al esfuerzo humano internacional para mejorar la calidad de vida tanto en estados Unidos como en México" (FUMEC, 2000). Además, los primeros programas e iniciativas de la FUMEC igualmente confirman esta característica filantrópica; en el reporte de actividades de los primeros cinco años de la fundación, las tres áreas prioritarias fueron el medio ambiente, la salud pública y los problemas socioeconómicos resultados de la integración de ambas sociedades (FUMEC, 1998). Actualmente la FUMEC cuenta con una estrategia que se centra en el desarrollo de proyectos específicos que responden a ventanas de oportunidad que han sido identificadas a través de estudios de prospectiva. Los proyectos que desarrollan en la actualidad están organizados en tres áreas estratégicas diferentes a las definidas en la creación de la fundación: a. desarrollo económico basado en la innovación tecnológica, b. desarrollo de recursos humanos y c. medio ambiente y salud. Este cambio o ajuste de áreas estratégicas constata la característica de desarrollar iniciativas en campos donde los conocimientos presentan altos grado de aplicación, característica que ya evocamos líneas más arriba.

Además, al momento de la creación de la FUMEC, este organismo limitaba su campo de acción a la zona fronteriza entre México y Estados Unidos. Sin embargo, el perfil de la FUMEC no tardo muchos años en cambiar, ya que esta fundación en efecto extendió su zona de acción a todo México, por una parte, y cambió como lo venimos de mencionar sus áreas estratégicas hacia campos con mayor impacto socioeconómico para América del Norte. Es en esta nueva lógica que la FUMEC define el desarrollo de los Micro Sistemas Electromecánicos (MEMS) y la industria aeroespacial y automotriz como campos prioritarios (FUMEC, 2010). 


\section{Financiamiento e independencia institucional}

Para financiar la creación de la FUMEC, una primera propuesta fue la del modelo debt-for-science swaps propuesto por Brown y Sarewitz (1991), que se trata de un modelo inspirado del debt-for-nature swaps ${ }^{39}$. Esta propuesta no fue concretada y finalmente el fondo económico que sustento la creación de la FUMEC fue constituido por partes iguales de los gobiernos de México y de Estados Unidos en 1992. Por parte de Estados Unidos, la Agencia de Desarrollo Internacional (AID) aportó 2 millones de dólares. Del lado mexicano, fue el Consejo Nacional de Ciencia y Tecnología (CONACYT) quien aportó la misma cantidad. Dos años más tarde, la AID destinó un monto más de 150000 dólares (FUMEC, 1998). Estas aportaciones constituyen un fondo propio del organismo, que es la base económica para asegurar su funcionamiento. De hecho, los gastos de operación para la FUMEC son financiados por los intereses obtenidos de la inversión en la banca de este fondo. Esta forma de operar económicamente le da a la FUMEC una independencia económica frente a las instituciones públicas y privadas. Es importante señalar que este fondo que representa el patrimonio de la fundación no ha dejado de aumentar, esto ya que paso de 4 millones de dólares en su creación a 13,9 millones de dólares a finales de 2009.

Por otra parte, los proyectos desarrollados por la fundación son financiados en su mayoría con recursos provenientes de instituciones públicas. La FUMEC retiene un porcentaje de estos financiamientos como organismo gestor. Estos recursos son bastante importantes, por ejemplo, López y colaboradores en un reporte sobre los Fondos PYMES de la Secretaria de Economía han identificado que la FUMEC fue en 2006 el organismo que más fondos gestionó en este programa gubernamental. En este documento, se reporta que entre el 2004 y el 2006 esta fundación recibió un total de 328.445 millones de pesos mexicanos (aproximadamente 29.8 millones de dólares) para financiar diferentes proyectos, entre los cuales se encuentra la tecnología de Microsistemas que es el caso que estudiamos.

\section{La Junta de Gobierno y su red política}

Dentro de la fundación las decisiones son tomadas por un órgano llamado "Junta de Gobierno" que esta conformado por 16 personas: 8 mexicanos y 8 estadounidenses. Estas personas son personalidades provenientes de la academia, de instituciones gubernamentales y de la industria. Se trata entonces de un grupo bastante heterogéneo que representa diversos intereses. A pesar del origen de estas personalidades, la FUMEC se presenta como un organismo independiente de las esferas institucionales gubernamentales, privadas y académicas. Esta etiqueta de "independiente",

\footnotetext{
${ }^{39}$ El modelo del debt-for-nature swaps consiste en retirar una parte de la deuda externa de un país en intercambio de su cooperación en materia de conservación de su propio medio ambiente (Gonzalez-Fernandez y Pérez-Ímigo, 2008).
} 
que re-envía al término "no gubernamental", confiere a la fundación una cierta legitimidad frente a otras instituciones, ya que la FUMEC no es percibida como un concurrente potencial. Esta legitimidad es confirmada durante una entrevista con un agente de la FUMEC, quien nos dice con respecto a una pregunta sobre la posición de la fundación con las demás instituciones:

“(...) Entonces, nosotros somos un ente que no pertenecemos a ningún sector y este carácter de neutralidad nos da mucho valor ante las instituciones, porque no representamos competencia para ninguno y en realidad nuestra función en promover $y$ es ayudar a que estos programas se realicen. (...)" (Avendaño, 2009).

Por otra parte, las personalidades de estos tres sectores que forman la Junta de Gobierno representan la base de una red de contactos en diversas instituciones públicas y privadas que son movilizados con fines de obtener alianzas e informaciones útiles para llevar a cabo los programas. Esta Red que calificamos como "red política", ya que esta fundada sobre relaciones políticas, constituye un capital importante para la fundación. Esta importancia se refleja en el hecho que la FUMEC ha logrado dar continuidad a sus programas con financiamiento público a pesar de la discontinuidad en las políticas en ciencia y tecnología, así como las de desarrollo económico, a través de los diferentes periodos presidenciales ${ }^{40}$. Esta importancia de la red política de la FUMEC y de su movilización se refleja en la siguiente citación del Presidente de la Junta de Gobierno que hemos extraído de uno de los reportes de actividades de la fundación:

"Otro esfuerzo estratégico mayor en el periodo fue renovar y fortalecer nuestra red de relaciones con las dos Agencias Federales clave para nuestra Misión, la Secretaria de Economía (SE) y el Consejo Nacional de Ciencia y Tecnología (CONACYT), una vez realizado el cambio de Administración Pública Federal en México, en 2006. Estas dos Agencias son tanto los contribuyentes mexicanos a nuestro Fondo Patrimonial, como los mayores clientes y patrocinadores de nuestros programas. Se consolidó una relación muy cercana y efectiva con los funcionarios clave de estas Agencias" (FUMEC, 2008).

\section{La FUMEC en la emergencia y el desarrollo de la Tecnología de Microsistemas en México}

\section{La emergencia de los MEMS}

MEMS es el acrónimo en inglés de "sistemas microelectromecánicos", que son dispositivos que tienen una longitud menor a $1 \mathrm{~mm}$ y mayor a $1 \mu \mathrm{m}$, que combinan componentes tanto eléctrico como mecánicos y que son fabricado usando tecnologías de procesos de circuitos integrados (Gad-el-Hak, 2001).

\footnotetext{
${ }^{40}$ La discontinuidad de las políticas en ciencia y tecnología como en otros ámbitos es una de las características del sistema político en México. Sobre este punto, Casas y Dettmer (2007) han señalado que esta discontinuidad es un obstáculo que impide la definición de un paradigma consistente en la política nacional en ciencia y tecnología.
} 
También se usa el término de Tecnología de Microsistemas para referirse a este tipo de dispositivos que son cada vez más presentes en la vida cotidiana. De hecho, esta presencia se debe a la gran cantidad de aplicaciones de estos dispositivos que se sustentan en los avances en la nanotecnología y en los materiales avanzados para aumentar la capacidad de miniaturizar componentes electrónicos y mecánicos. Es por esto que los avances de la microtecnología están estrechamente ligados a los avances científicos y tecnológicos en la escala nanométrica. Unas de las primeras aplicaciones de estos dispositivos fueron los acelerómetros integrados en los sistemas de seguridad de los automóviles que activan las bolsas de aire al momento de las coaliciones. Otro ejemplo lo encontramos en los teléfonos portátiles o cámaras fotográficas que al girarlos sobre el plano horizontal la visualización de las pantallas permanece estable en este plano.

En la literatura académica los primeros artículos científicos datan de la década de 1960 (Flores-Herrera, 2007). La fabricación de los primeros dispositivos MEMS apareció en la década de 1970, sin embargo, los primeros MEMS en el mercado aparecieron en la década de 1980 (las bolsas de aire en los automóviles). Actualmente existen centros públicos y privados de investigación y desarrollo de estos dispositivos principalmente en los países que lideran los avances tecnológicos, estando uno de los centros más importante en el Estado de Nuevo México, en Estados Unidos. Se trata de los Laboratorios Sandia, que es un centro de investigación especializado en el armamento que ya en el 2000 contaba con un total de 500 investigadores e ingenieros trabajando en el desarrollo de MEMS (Freiburghouse, 2001). Además, en el ámbito académico la progresión de estas tecnologías se refleja en la proliferación de revistas especializadas en este campo que surgió a partir de las década de 1990, así como en el crecimiento de las publicaciones en este campo (Vinck y Robles-Belmont, 2011).

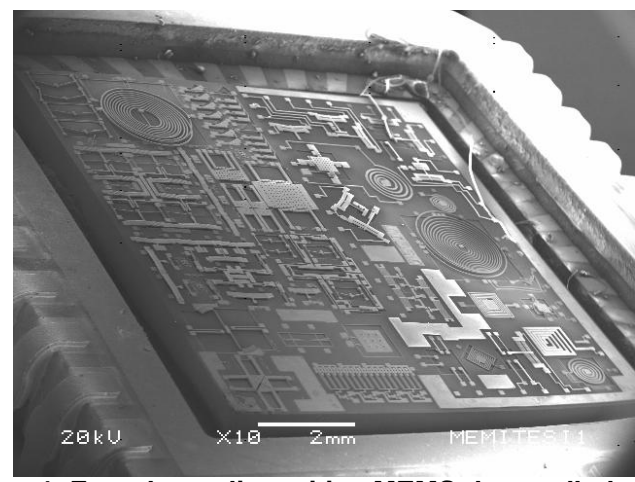

Imagen 1. Foto de un dispositivo MEMS desarrollado en el Instituto Tecnológico Superior de Irapuato y el Centro de Física Aplicada y Tecnología Avanzada de la UNAM. Fuente: http://www.victorcastano.net/5.html

Fuera del mundo académico, las publicaciones sobre la Tecnología de Microsistemas también han conocido un crecimiento importante, se trata 
de hecho de revistas sobre las actualidades del desarrollo de estas tecnologías. Algunas de estas revistas son el canal de comunicación de empresas dedicadas a la vigilancia tecnológica, que proponen reportes y estudios de prospectiva y de mercado en torno al desarrollo de los MEMS.

El desarrollo de estas tecnologías se concentra principalmente en Estados Unidos. China, Japón y los países de la Comunidad Europea son los países donde el desarrollo de los MEMS es también importante. En América Latina, el país donde más avances hay en este campo es Brasil. México ocupa el segundo lugar en esta última región. La emergencia de estas tecnologías es prácticamente reciente en México, ya que en un estudio cienciométrico se ha mostrado que las primeras publicaciones datan a partir de 2002 y hasta el 2009 la producción de artículos fue de 25 documentos, lo que se traduce como una tecnología en estado embrionario en México (Robles-Belmont, 2011).

\section{El programa de Microsistemas de la FUMEC}

El análisis de publicaciones científicas muestra que la tecnología de microsistemas en México esta en sus primeras etapas de emergencia. Actualmente, en México al menos 14 centros e instituciones de investigación y de educación desarrollan actividades para el desarrollo de estas nuevas tecnologías. Estas actividades están orientadas hacia la formación de recursos humanos especializados, la instalación de nuevas infraestructuras científicas y tecnológicas, así como el desarrollo de proyectos de diseño, de fabricación y de caracterización de prototipos de dispositivos MEMS. Durante nuestra encuesta, identificamos que muchas de estas iniciativas se inscriben en el "Programa de Microsistemas" de la FUMEC.

Este programa de la FUMEC para el desarrollo de MEMS inició en el año 2000 durante una serie de talleres sobre la tecnología de los MEMS organizado por la misma fundación, los Laboratorios Sandia y la Universidad de Texas en El Paso (FUMEC, 2002). Fue en este taller que la tecnología de los MEMS fue identificada como una ventana de oportunidad para la zona fronteriza entre México y Estados Unidos. El análisis del contexto en esta zona indicó la presencia de empresas basadas en estas tecnologías del lado de Estados Unidos, pero también se señaló la falta de capacidades científicas y tecnológicas del lado mexicano. Sin embargo, esta carencia no represento un obstáculo mayor para la FUMEC, ya que la fundación convencida de las potencialidades de estas tecnologías definió un programa dedicado al desarrollo de los MEMS. Al inicio, dos retos fueron definidos dentro del marco de este programa: formar recursos humanos especializados en el campo de los MEMS y construir infraestructuras científicas y tecnológicas para el desarrollo de dispositivos.

Este programa esta formado por diversas iniciativas, y en su evolución nosotros distinguimos dos fases. En la primera fase, identificamos dos iniciativas orientadas a cubrir la falta de recursos humanos y de infraestructura. Una de estas iniciativas, la primera, fue lanzada en el 2003 
con el fin de instalar infraestructura para la concepción de dispositivos MEMS; se trata de la Red Nacional de Centros de Diseño de MEMS (CDMEMS). Paralelamente a la creación de esta red, la FUMEC coordinó también la integración de un grupo de investigadores especialistas con el fin de desarrollar la formación de recursos humanos y de promover el desarrollo de esta tecnología en México. Más tarde, en el 2004, con el fin de abarcar las demás etapas del desarrollo de dispositivos MEMS, la FUMEC promovió y apoyó la construcción de tres laboratorios para la fabricación y la caracterización de estos dispositivos. Esta segunda iniciativa enfocada también a la infraestructura lleva el nombre de Red de Laboratorio de Innovación de MEMS (LI-MEMS).

La segunda fase que distinguimos en el Programa de Microsistemas de la FUMEC esta constituida por tres iniciativas orientadas a la integración de los diversos sectores potenciales para el desarrollo de los MEMS y a la comercialización de estos dispositivos. La primera iniciativa fue la creación del Centro de Articulación Productiva en MEMS (CAP-MEMS) en el 2004. Un año más tarde se creó el Consorcio Mexicano de Microsistemas (CMM). La tercera iniciativa es la constitución de la Alianza Estratégica y Redes de Innovación en MEMS (AERI-MEMS), creada en el 2008 dentro del marco de un programa del CONACYT para formar redes nacionales de innovación en áreas estratégicas.

Estas cinco iniciativas dan forma a la emergencia de la tecnología de microsistemas en México. Durante nuestra encuesta hemos notado que estas iniciativas están ligadas la unas con las otras. Estas relaciones están basadas en los ajustes que el Programa de Microsistemas de la FUMEC ha conocido a los largo del desarrollo de cada iniciativa. Por ejemplo, las bases para crear el CMM fueron el CAP-MEMS, el CD-MEMS y el LI-MEMS. Además de estas iniciativas, la FUMEC llevó a cabo otras acciones paralelamente para la promoción de las iniciativas mismas, así como la difusión del desarrollo de los MEMS y los usos potenciales de esta tecnología. Estas actividades desarrolladas en espacios académicos e industriales le permitieron a la fundación identificar nuevos actores científicos y económicos potenciales para el desarrollo de estas nuevas tecnologías. Las iniciativas que hemos evocado más arriba son representadas en la siguiente imagen y en los siguientes puntos las detallaremos. 


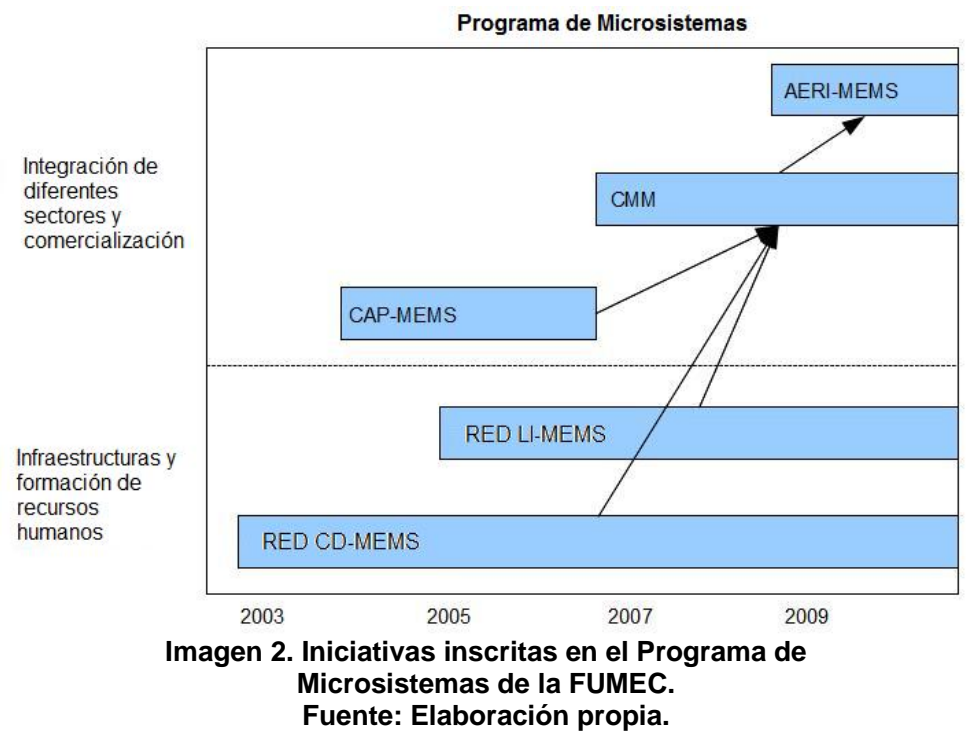

\section{Instalación de infraestructuras científicas y tecnológicas en México}

Para desarrollar este tipo de tecnologías se requieren de recursos onerosos para la instalación de infraestructuras científicas y tecnológicas, para la formación de recursos humanos y para el desarrollo de proyectos de investigación y desarrollo de dispositivos MEMS. El financiamiento para la instalación de estas infraestructuras es tradicionalmente financiado por el Estado, y esto a través de políticas orientadas hacia el desarrollo de nuevas ciencias y tecnologías. Sin embargo, para el caso mexicano, ya hemos mencionado que no se cuenta con un Programa o Plan Nacional para el desarrollo de las MNT. A pesar de esto, las dos iniciativas para la instalación de infraestructuras fueron desarrolladas gracias al financiamiento público gestionado por la FUMEC.

Un primer paso fue el de llamar la atención de los decidores políticos hacia el desarrollo de los MEMS; esto sucedió en el 2003 durante un evento organizado por la FUMEC, el CONACYT y la empresa transnacional AMD, al cual asistieron el Presidente de México y el Secretario de Economía, quienes mostraron un interés particular por el desarrollo de la tecnología de microsistemas. En ese mismo año, una serie de reuniones con funcionarios de la Secretaría de Economía (SE) tuvieron lugar y el resultado fue el proyecto de la creación de la Red CD-MEMS. La convocatoria tuvo una respuesta bastante amplia ya que un total de 12 entidades académicas respondieron, de las cuales sólo 10 lograron concretizar e instalar las infraestructuras. Es importante mencionar, que el financiamiento de estos centros de diseño no fue cubierto en su totalidad por la SE, la participación de las instituciones donde fueron instalados fue uno de los requisitos en la convocatoria. La parte cubierta por la SE se centro en los equipos de computo y en los programas para la concepción y 
simulación, la adecuación de los locales donde fueron instalados los CDMEMS fue cubierta por las instituciones académicas.

Estos diez centros están distribuidos principalmente en la zona del centro, del Bajío y del norte del país. En el centro-sur del país cinco CDMEMS han sido instalados: en la Ciudad de México dentro de la Faculta de Ingeniería de la Universidad Nacional Autónoma de México (UNAM); en el Estado de Puebla en el Instituto Nacional de Astrofísica, Óptica y Electrónica (INAOE) y en la Universidad Popular Autónoma del Estado de Puebla (UPAEP); en el Estado de Morelos en el Instituto de Investigaciones Eléctricas; y en Veracruz dentro de la Facultad de Ingeniería de la Universidad Veracruzana. Tres centros más fueron instalados en la región del Bajío: uno en el Centro de Investigaciones y Estudios Avanzados (CINVESTAV) campus Guadalajara; el segundo en la Universidad de Guadalajara, campus Ciénega; y el tercer centro en esta región en el Instituto de Estudios Superiores de Irapuato, en el Estado de Michoacán.

En la región del norte del país se instalaron dos CD-MEMS, ambos en la zona fronteriza con Estados Unidos. El primer CD-MEMS fue instalado en la Universidad Autónoma de Ciudad Juárez (AUCJ) y el segundo centro en el Instituto tecnológico de Estudios Superiores de Monterrey (ITESM). Aquí es importante señalar que al inicio el Programa de Microsistemas de la FUMEC sólo contemplaba impulsar el desarrollo de esta tecnología en la zona fronteriza, pero la respuesta a la convocatoria fue también por parte de entidades en otras regiones del país. Además, llevar la iniciativa fuera de la zona fronteriza fue una de las condiciones de la SE para apoyar la iniciativa de la Red CD-MEMS.

La segunda iniciativa orientada a la instalación de infraestructuras fue lanzada una vez que la Red CD-MEMS se consolido. En ese momento la FUMEC extendió su estrategia sobre las demás etapas del desarrollo de MEMS: la fabricación y la caracterización. El objetivo principal de esta segunda red es poner a disposición la infraestructura necesaria para la fabricación con aplicaciones específicas en tres nichos de oportunidad: BioMEMS, MEMS para la industria automotriz y MEMS para las telecomunicaciones. Aquí es interesante resaltar que estas tres ventanas de oportunidad fueron identificadas por la FUMEC a partir de estudios de prospectiva y de vigilancia tecnológica elaborados por otros organismos, entre los cuales figura la Fundación de Educación y Comercialización de la Micro y Nanotecnología (MANCEF por sus siglas en inglés).

También con el apoyo de la SE la FUMEC lanzó en 2005 la iniciativa para crear la Red LI-MEMS. El primer laboratorio fue construido en las instalaciones del Instituto Nacional de Astrofísica, Óptica y Electrónica (INAOE) en el Estado de Puebla. Este laboratorio es de hecho parte de un proyecto más amplio en este instituto Ilamado Laboratorio Nacional de Nanoelectrónica (LNN), proyecto en el cual además de participar la SE y la FUMEC, la Secretaria de Desarrollo Económico del Estado de Puebla también es presente. Esta presencia del gobierno local se debe a que en la ciudad de Puebla se encuentra una planta de la Volkswagen que genera importantes ingresos económicos para la región, lo que ha hecho que parte 
de la política económica de la región sea impulsar el sector automotriz que es un importante consumidor de dispositivos MEMS. En este laboratorio ha sido instalada la infraestructura científica y tecnológica necesaria para fabricar ciertos prototipos de MEMS basados en la microelectrónica. De hecho, la iniciativa del LNN surge dentro del INAOE a partir de la necesidad de renovar la infraestructura del laboratorio de microelectrónica de este instituto que existe desde los años 70. Además, parte de esta infraestructura proviene de una donación por parte de la empresa Motorola efectuada en el marco de la iniciativa llamada LatinChip pocos años antes.

El segundo laboratorio ha sido instalado en la Facultad de Ingeniería de la Universidad Nacional Autónoma de México (UNAM) y la infraestructura disponible es dedicada a la fabricación y la caracterización de dispositivos MEMS. El LI-MEMS y el CD-MEMS instalados en esta universidad se inscriben en un proyecto más amplio llamado UNAMems. Se trata de un centro de desarrollo de MEMS que desarrolla actividades para el desarrollo de antenas RFID, sensores basados en fibra óptica, MEMS para sistemas de resonancia magnética y BioMEMS para aplicaciones medicas. La construcción de este centro ha sido financiada por la SE (vía las iniciativas CD-MEMS y LI-MEMS) y la UNAM. Actualmente en el centro UNAMems tiene capacidades para la concepción, la simulación y la fabricación de dispositivos MEMS, y este centro pretende orientar sus esfuerzos de investigación hacia el desarrollo de MEMS basado en materiales biocompatibles que son más económicos.

El tercer laboratorio de la Red LI-MEMS ha sido instalado en la Universidad Autónoma de Ciudad Juárez (UACJ), en la zona fronteriza entre México y Estados Unidos. Este laboratorio esta localizado a proximidad de los principales centros de desarrollo de MEMS en Estados Unidos y en una zona donde las actividades económicas en torno a la fabricación de MEMS son bastante importantes, principalmente la industria maquiladora automotriz. La infraestructura fue instalada en este LI-MEMS principalmente para el encapsulado de dispositivos MEMS. Este laboratorio que también fue apoyado por el gobierno local y, junto con el CD-MEMS en esta universidad, forma parte del Centro de Investigación en Ciencia y Tecnología Aplicada (CICTA). Actualmente, en este centro se cuenta con capacidades para cubrir todas las etapas para el desarrollo de los MEMS.

La construcción de los CD-MEMS y LI-MEMS constituyen entonces las infraestructuras de base para el desarrollo de MEMS en México. Estas infraestructuras además sirven para la formación de recursos humanos en este campo, este punto lo abordaremos más adelante. Sobre las infraestructuras, otro punto importante para el desarrollo de MEMS es la producción en masa de estos dispositivos. La FUMEC también ha centrado esfuerzos en la producción en masa de MEMS, sin embargo, ninguno de los laboratorios mencionados esta equipado para esto. El laboratorio que tiene mayor capacidad es el INAOE, donde de hecho la segunda etapa del LNN, aún en desarrollo, comprende un laboratorio dedicado a la fabricación a pequeña de dispositivos MEMS. 
La organización de las actividades de investigación y desarrollo en torno a los MEMS bajo la forma de redes de colaboración ha sido promovida por la FUMEC, esto con el objetivo de hacer frente a la insuficiencia de recursos materiales disponibles. Se trata en efecto de racionalizar los pocos recursos disponibles poniéndolos en común entre toda la comunidad científica. Este fenómeno en la constitución y reorientación de redes científicas en el desarrollo de las MNT ya ha sido analizado en un trabajo previo (Robles-Belmont, 2009). Otro factor que influyó fue la condición de la SE de la "no duplicidad" de infraestructura dentro del marco de los LIMEMS. De hecho, es por este motivo que cada uno de los tres LI-MEMS esta especializado en etapas diferentes y técnicas para el desarrollo de MEMS.

En el proceso de instalación de las infraestructuras para el desarrollo de la tecnología de microsistemas en México, las funciones que la FUMEC ha desempeñado son la de obtener los recursos financieros y la de gestionar los proyectos para la construcción de los laboratorios. Dentro de este proceso, la red de política de la FUMEC ha jugado un papel importante para contactar, convencer y entablar las negociaciones para el apoyo del desarrollo con los decidores políticos, donde la FUMEC ha movilizado informaciones sobre las potencialidades económicas y sociales de las microtecnologías. También, por otra parte, las particularidades de las dinámicas económicas y sociales de los contextos locales donde han sido instalados los laboratorios han sido consideradas por la fundación para la definición del Programa de Microsistemas y su justificación. Aquí es a través de la articulación de lo global con lo local que la FUMEC logra llamar la atención de actores económicos y políticos claves para lograr las primeras etapas para el desarrollo de los microsistemas en México, y esto sin que exista en este país una política explicita para este tipo de tecnologías.

\section{Formación de recursos humanos para el desarrollo de la microtecnología en México}

Recordemos que al inicio del Programa de Microsistemas, la FUMEC había identificado que uno de los retos a cubrir era la formación de especialistas. Sin embargo, la formación y acumulación de recursos humanos especialistas en este campo no fue objeto de iniciativas como las de la creación de redes científicas. La formación de recursos humanos fue llevada a cabo de forma transversal dentro de las diferentes iniciativas del Programa de Microsistemas. Dentro del marco de este programa, las acciones y las actividades identificadas para paliar la falta de recursos humanos se ha desarrollado principalmente en dos niveles: la formación científica y la formación profesional.

Por parte de la formación científica, las primeras acciones tuvieron lugar en el contexto de la creación de la Red CD-MEMS. Dentro del proyecto de la creación de esta red, en el 2002, un programa de especialización sobre la tecnología MEMS fue efectuado por la FUMEC. Este programa de formación de especialistas consistió en una serie de 
cursos y de talleres sobre estas tecnologías que fueron impartidos a investigadores de las instituciones retenidas en la convocatoria de esta red. Esta primera formación de especialistas fue impartida por investigadores provenientes de las principales universidades estadounidenses en el campo de los MEMS: Universidad de Texas y los Laboratorios Sandia. Estas iniciativas de formación científica se centraron sobre aspectos de diferentes técnicas teóricas para la fabricación de MEMS y sobre la concepción y la simulación asistida por computadora. Con el fin de promover los CD-MEMS y de promover la colaboración entre los diferentes participantes científicos, los cursos y talleres fueron impartidos en las diferentes instituciones que acogieron los CD-MEMS y en cuatro ocasiones en las instituciones en Estados Unidos de donde provenían los formadores.

Posteriormente, dentro de la iniciativa del CAP-MEMS, estas formaciones para los científicos continuaron. El proyecto que fue financiado por la SE con el fin de reforzar las capacidades del CAP-MEMS de la FUMEC. En este segundo caso, se trato de cursos técnicos propuestos a los miembros de las dos redes y de otras instituciones educativas y de investigación no participantes. En el marco del CAP-MEMS, identificamos cinco cursos y talleres llevados a cabo en el 2004 (ver cuadro 1.) que reflejan la dinámica de la formación de especialistas en MEMS dentro del programa de la fundación.

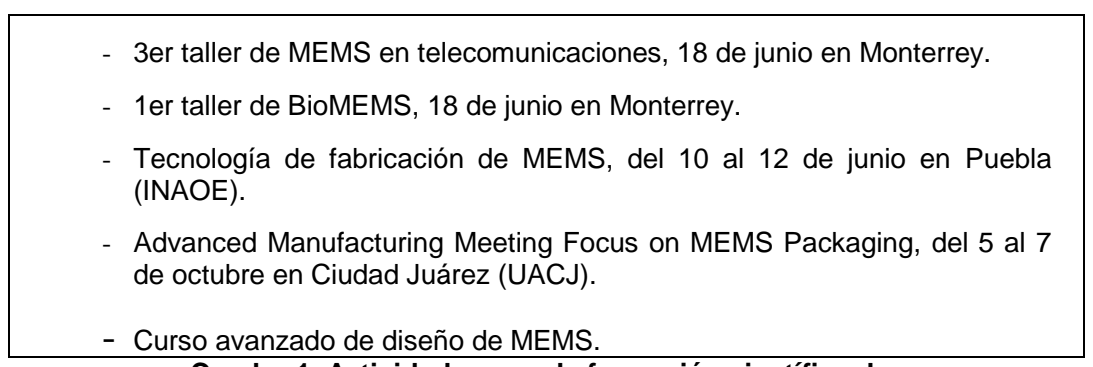

Cuadro 1. Actividades para la formación científica de especialistas en MEMS en México dentro del marco del CAP-MEMS de la FUMEC.

Por otra parte, este tipo de cursos de formación de investigadores tuvieron lugar en eventos académicos y profesionales en el campo de los MEMS. Por ejemplo, tal fue el caso del curso "MEMS -CAD course- Coventor \& SoftMEMS" organizado por la FUMEC en el marco de las actividades de la conferencia COMS $2008^{41}$ en México. En las iniciativas que siguieron a las de las redes y del CAP-MEMS, también se continuaron las actividades para la formación de especialistas en MEMS. En la última iniciativa de la FUMEC, el CMM, uno de los objetivos es "la formación y el refuerzo de recursos

\footnotetext{
${ }^{41}$ En 2008, el CMM de la FUMEC con coordinación con la fundación MANCEF, el Consejo de Ciencia y Tecnología del Estado de Jalisco (COECYT-Jalisco) y la Cámara Nacional de la Industria de la Electrónica y de las Tecnologías de la Información (CANIETI) organizaron la conferencia internacional COMS en Puerto Vallarta, México, que tiene como principal objetivo reunir a los sectores científicos y económicos implicados en el campo de la tecnología MEMS.
} 
humanos de alto nivel". Es entonces en esta marco que en el 2010 un curso sobre técnicas de diseño de dispositivos FPGA fue organizado por la FUMEC con el apoyo de la SE.

Sobre la formación orientada a profesionales que ha tenido lugar dentro de las iniciativas de la FUMEC para el desarrollo de la tecnología MEMS, una parte de este tipo de formaciones se lleva a cabo en los institutos de educación y de investigación donde han sido instalados los laboratorios CD-MEMS y LI-MEMS. Las infraestructuras instaladas sirven para las prácticas de laboratorio de diversos cursos de estudios en ingeniería que han incluido en sus programas asignaturas en torno al desarrollo de MEMS. Por otra parte, en algunas de las formaciones propuestas por la FUMEC han participados profesionales que ya trabajan en la industria. Tales son los casos de seis personas que han sido beneficiadas de becas puntuales para tener la formación por parte de la FUMEC sobre el diseño de dispositivos FPGA que venimos de evocar. Otro ejemplo es el taller sobre empaquetamiento de MEMS organizado en el 2004 en Ciudad Juárez con la empresa Delphi.

Las formaciones de recursos humanos cubiertas por la FUMEC no conciernen solamente aspectos técnicos, ya que también formaciones sobre temas de administración han sido propuestas dentro del marco del Programa de Microsistemas. Este tipo de formaciones profesionales han tenido lugar en las diferentes instituciones participantes en las dos redes de MEMS. Una de estas formaciones fue organizada, por ejemplo, por la UPAEP en el 2005 bajo la forma de un diplomado dirigido tanto a investigadores y estudiantes como a directivos de empresas e ingenieros. El título del diplomado fue: Capacitación Empresarial sobre Tecnologías MEMS. Esta formación se desarrollo durante varias semanas y tenia como objetivos específicos informar a los participantes sobre el desarrollo y las aplicaciones de los dispositivos MEMS, sobre la comercialización y la protección de las innovaciones en este campo, así como sobre el análisis de oportunidades para empresas mexicanas en el desarrollo de estas tecnologías. Los formadores en el diplomado fueron investigadores en el área de los MEMS mexicanos, y algunos provenientes de la Universidad de Texas y de la MANCEF. Este seminario recibió el apoyo económico de la SE y de la FUMEC. Otro ejemplo de este tipo de formaciones, es un taller que fue recientemente organizado por la FUMEC sobre las estas tecnologías en el marco del evento MexEEdev $2010^{42}$ en la Universidad Panamericana, en la Ciudad de México. Formación que tuvo el objetivo de transmitir las oportunidades de negocios que tienen las empresas mexicanas mediante el uso de las microtecnologías.

Los ejemplos que hemos evocado sobre las formaciones de recursos humanos en el marco del Programa de Microsistemas muestran que la FUMEC desempeña funciones en el financiamiento $y$ en la

\footnotetext{
${ }^{42}$ El MexEEdev es un Foro que se realiza al menos una vez al año en México dedicado a desarrolladores de sistemas electrónicos y programas embebidos (FPGA), que se trata de tecnologías estrechamente ligadas a los MEMS. De hecho, el Programa de Microtecnologías de la FUMEC comprende estas tecnologías.
} 
organización de actividades orientadas a la formación de especialistas científicos y profesionales. Las formaciones propuestas e impulsadas por la FUMEC abarcan todas las etapas para asegurar la instalación y el desarrollo de la tecnología MEMS en México. Otra función que hemos identificado por parte de la FUMEC en la formación de recursos humanos es la movilización de especialistas en el campo de las microtecnologías. Se trata del co-financiamiento con las demás instituciones gubernamentales participantes de la movilidad de especialistas provenientes principalmente de Estados Unidos para transmitir conocimiento de punta sobre el desarrollo de MEMS a investigadores mexicanos. También en algunos casos la FUMEC destino fondos para financiar estancias de estudiantes de doctorado o investigadores en el extranjero dentro del campo de las MNT.

\section{Promoción, diseminación y comercialización de la Tecnología de Microsistemas}

A lo largo de las iniciativas del Programa de Microsistemas, la FUMEC desarrollo diversas actividades encaminadas a la promoción, la divulgación y la comercialización de la tecnología MEMS en México y en el mundo. Estas actividades al igual que en el caso de la formación de recursos humanos han sido transversales en los diferentes proyectos e iniciativas.

La promoción para el desarrollo de las MNT (entre las cuales las MEMS) se ha llevado a cabo en prácticamente todos los foros académicos y profesionales donde la FUMEC participa como organizador y como invitado para presentar su programa para el desarrollo de MEMS en México. Desde inicios de este siglo, el desarrollo de las MNT fue una de las áreas estratégicas de la FUMEC. La fundación optó por el desarrollo de los MEMS a partir de una reunión con investigadores especialistas en las MNT de Estados Unidos, y esto a partir de las potencialidades técnicas y económicas que se han anunciado con la emergencia de estas nuevas tecnologías. En las presentaciones que utilizan los agentes de la fundación en los eventos donde participan encontramos los argumentos de estas potencialidades, principalmente las económicas. Como ya hemos mencionado anteriormente, la FUMEC inició con el Programa de Microsistemas solamente en la zona fronteriza, y rápidamente se extendió sobre el resto del territorio mexicano, esto al parecer porque el desarrollo de estas tecnologías se inserta en la carrera por el desarrollo de las nanotecnologías, que son percibidas como una oportunidad para sobrepasar el umbral del subdesarrollo.

No es evidente seguir la diseminación de las nuevas tecnologías. Durante nuestra investigación sobre el desarrollo de las MNT en México, un caso llamo nuestra atención. Se trata de una pequeña empresa productora de calzado especializado que tiene una línea de zapatos para diabéticos. La empresa presentó en un foro académico en torno a las nanotecnologías en México en el 2008 su interés en innovar estos zapatos al introducir 
nanopartículas de plata en el forro con el fin de evitar infecciones en los pies de las personas que presentan esta enfermedad ${ }^{43}$. La idea inicial de aplicar la nanotecnología en los zapatos surgió en una plática impartida por la FUMEC sobre el desarrollo de la nanotecnología algunos meses antes. La persona encargada del proyecto de los zapatos para diabéticos asistió a este evento y quedando convencida de las aplicaciones técnicas de la nanotecnología efectuó su presentación en el 2008, lo que llamo la atención de investigadores en el campo de la nanotecnología. La empresa junto con investigadores de diferentes centros de investigación ha desarrollado actividades de investigación financiadas por el CONACYT y sin la participación de la FUMEC.

Concerniente al desarrollo de los microsistemas, hemos identificado que se imparten cursos sobre los MEMS en diferentes institutos de educación que no han participado en las iniciativas de la FUMEC. Uno de estos casos es el laboratorio de Microtecnología y Sistemas Embebidos (MICROSE) del Instituto Politécnico Nacional, que es un laboratorio donde actualmente se llevan acabo actividades sobre los MEMS y la formación de recursos humanos en este campo. En una entrevista, el director de este laboratorio nos explica que la reorientación de las líneas de investigación de este laboratorio hacia el desarrollo de los MEMS ocurre en un contexto en el cual la emergencia de los microsistemas en México estaba en pleno desarrollo y los discursos se centraban sobre la promoción del desarrollo de estas tecnologías como una ventana de oportunidad para el país. En la integración de los MEMS como línea de investigación en este laboratorio, un agente importante fue el mismo director, quien ya estaba familiarizado con la tecnología de los MEMS ya que había participado al menos en una actividad en el Instituto Mexicano del Petróleo organizada por la FUMEC. De hecho, durante la entrevista el director de este laboratorio hace referencia a la FUMEC como el organismo que ha estado impulsando desde el inicio el desarrollo de la tecnología de microsistemas en México.

Estos dos casos de estudio los movilizamos en este texto para intentar dar cuenta la diseminación de nuevas tecnologías. Nos parece interesante ya que en la teoría sobre los cambios tecnológicos y los sistemas de innovación es ampliamente reconocido que la diseminación de la tecnología pasa por el flujo de informaciones entre los diferentes implicados (OCDE, 1997) y que estos cambios tecnológicas suceden en procesos discontinuos (Pérez, 2004). En ambos casos se confirma el flujo de información entre los implicados y la complejidad de los procesos de cambio tecnológico, donde la FUMEC ha desempeñado las funciones de producir ciertas informaciones y transmitirlas. En algunos casos, las funciones ligadas a la diseminación de esta nueva tecnología la calificamos como "función latente" (Merton, 1949), ya que no se trata de una función no buscada por parte de la FUMEC.

\footnotetext{
${ }^{43}$ Una cuarta parte de las personas que presentan esta enfermedad tienen complicaciones por pie diabético, que son complicaciones por infecciones desarrolladas en los pies que pueden llevar a la amputación de las extremidades (Bogdanchikova et al., 2009).
} 
Sobre la comercialización de los productos basados en la tecnología MEMS, ya hemos evocado de las iniciativas del CAP-MEMS y del CMM tienen dentro de sus objetivos este punto. EI CAP-MEMS que fue creado en el 2004, es un spin-off de la FUMEC que tiene como objetivo principal "facilitar vínculos entre empresarios, académicos y tomadores de decisiones con el fin de general colaboraciones que permitan el desarrollo de nuevos productos y negocios" (FUMEC, 2006). Para el caso del CMM, en uno de sus objetivos se especifica la "asistencia y promoción de cursos, diplomados, foros y eventos especializados de prospectiva y gestión tecnológica, protección intelectual, negocios de alta tecnología, comercialización, etc.".

Otra iniciativa orientada a la comercialización de productos basados en la tecnología MEMS es la AERI-MEMS creada en el 2008. Ya mencionamos que esta iniciativa liderada por la FUMEC se inscribe en un programa del CONACYT. Dentro de esta iniciativa se llevaron a cabo acciones para vincular a entidades principalmente del sector productivo y académico, con el fin de desarrollar proyectos de aplicaciones de MEMS en los sectores de la salud, de los alimentos, de lo automotriz y de la energía. En los proyectos que se han apoyado dentro del marco de esta iniciativa es un requisito la participación de empresas en torno a productos. Un total de tres proyectos de aplicaciones han podido ser concretizados en este marco, el primero es sobre una videocámara $3 \mathrm{D}$ actualmente comercializada y que ha sido desarrollada por la empresa Prefixa. La FUMEC apoyó y siguió a esta pequeña empresa para presentar el proyecto de la videocámara ante el CONACYT y la SE, para financiar la fabricación. El segundo proyecto es el desarrollo conjunto entre la UACJ y la empresa Team Technologies de un interruptor basado en la tecnología RF-MEMS con aplicaciones para los teléfonos portátiles. Una patente ha sido generada y el proyecto ha sido financiado parcialmente por el CONACYT. Team Technologies es actualmente catalogada como una empresa de base tecnológica que cuenta con capacidades de innovación importantes que han sido adquiridas gracias a financiamientos públicos obtenidos con el apoyo y seguimiento de la FUMEC. El tercer proyectote innovación es el desarrollo de un sistemas biomédico basado en MEMS para el monitoreo del flujo respiratorio de neonatos. Se trata de un proyecto desarrollado por la pequeña empresa de base tecnológica Biomedical Integral, que es especializada en la fabricación y comercialización de incubadoras para prenatales, el financiamiento proviene del CONACYT y la concepción de los MEMS es asegurada por la UACJ, la FUMEC ha apoyado la constitución y seguimiento del proyecto.

\section{Discusión y conclusiones}

A lo largo de este artículo hemos trazado la emergencia y el desarrollo de la tecnología de MEMS en México desde inicios de este siglo, donde hemos mostrado que la FUMEC ha sido el principal impulsor del desarrollo de estas nuevas tecnologías. Igualmente confirmamos que la emergencia de los 
MEMS ha tenido sus orígenes en las iniciativas llevadas a cabo en diferentes etapas y niveles por este organismo.

De un lado, la caracterización y la calificación de la FUMEC nos aportan elementos para mejor comprender el lugar que ha ocupado y ocupa este organismo, que calificamos con orígenes "filantrópicos", en las dinámicas de la instalación, del desarrollo y de la diseminación de las MNT en México. En esta primera parte, vimos que la conformación de la Junta de Gobierno de la fundación le confiere un lugar importante a este organismo. En efecto, lo que hemos caracterizado como "red política" le permite a la fundación una cierta movilidad a través de las otras esferas institucionales para la formulación de sus programas y para el desarrollo de éstos sin ser demasiado afectados por los cambios del poder político en el país. Esta posición que ocupa la FUMEC se da en los diferentes niveles de gobierno (local, regional y nacional), y en conjunto con las informaciones que produce y adquiere, la fundación logra tener un conocimiento de los contextos locales y regionales. Esto le permite a este organismo tener influencias en los sistemas científicos y tecnológicos en los diferentes niveles en el país, ya sea al participar en las convocatorias con sus propios proyectos 0 al desempeñar funciones como miembro de los comités evaluadores.

Con respecto a las funciones desempeñadas por los organismos filantrópicos y no gubernamentales en los cambios científicos y tecnológicos, en la literatura académica que presentamos brevemente en este artículo se distinguen al menos siete funciones: 1. Financiamiento de actividades de la investigación, 2. Instalación de infraestructuras, 3. Promoción de nuevas ciencias y tecnologías, 4. Aplicación de nuevas tecnologías, 5. Creación de redes científicas, 6. Formulación de políticas y 7. Cambios de modelos institucionales. En el análisis del Programa de Microsistemas de la FUMEC, hemos identificado que este organismo ha llevado a cabo actividades en estas funciones. Además, identificamos que ha desempeñado funciones centrales en la formación de recursos humanos, así como en la producción y en el flujo de informaciones sobre las nuevas tecnologías para su diseminación y comercialización. El estudio de la instalación de infraestructuras, de la organización de redes científicas y tecnológicas, de la formación de recursos humanos, así como de la promoción, de la diseminación y de la comercialización de las tecnologías de MEMS, nos aportan elementos que nos permiten dar cuenta de las nuevas dinámicas en el desarrollo de nuevas ciencias y tecnologías. Donde organismos diferentes a los tradicionalmente estudiados están llevando a cabe funciones que anteriormente fueron desempeñadas por otros organismos de las otras esferas institucionales. Estos cambios en las funciones de las diferentes esferas institucionales, llaman a desarrollar otros estudios empíricos en torno a estas nuevas dinámicas.

Por otra parte, el caso de estudio de la FUMEC y el desarrollo de los MEMS en México, muestra como este tipo de organismos, que no entra en las esferas institucionales de la academia, del gobierno y de la industria, pueden desempeñar funciones en los procesos de instalación y de desarrollo de nuevas tecnologías. Lo anterior, como lo mencionamos en la 
parte introductiva del artículo, pone en cuestión la modelización de las relaciones entre los diferentes organismos que han sido propuestos en la literatura académica, modelos que son comúnmente representados por un triángulo. Además, evocamos que la presencia de estos organismos, que calificamos como "filantrópicos" y "no gubernamentales", ya ha sido abordada en diferentes investigaciones en las ciencias sociales. Es a partir de nuestras observaciones sobre el caso de los MEMS en México y apoyados en las otras investigaciones sobre estos organismos que proponemos extender el abanico de entidades a tomar en cuenta en este tipo de estudios. Para incluir a estos organismos como una cuarta esfera en los modelos de las relaciones proponemos la figura del tetraedro, donde cada vértice representa a cada una de las esferas y las aristas representan las relaciones posibles en los procesos de la producción, del uso y de la diseminación de nuevos conocimientos (ver imagen 3). Cabe mencionar que esta representación de las relaciones no significa que es necesario reunir las cuatro esferas institucionales para lograr la producción y aplicación de nuevos conocimientos.

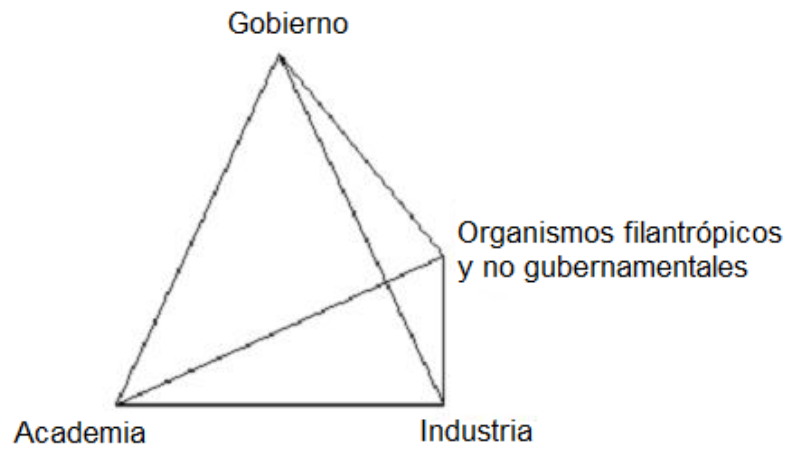

Imagen 3. Tetraedro de las posibles relaciones en la producción, el uso y la diseminación de nuevos conocimientos.

Además, hacemos énfasis en la implicación política de los modelos de la producción y de la aplicación de nuevos conocimientos, ya que estos modelos teóricos a la base son traducidos por los practicantes (consejeros, consultores, políticos, etc.) como modelos para el desarrollo tecnológico a reproducir. Esto tiene evidentemente fuertes implicaciones económicas y sociales, ya que a partir de estos modelos se definen políticas en ciencia y tecnología que están estrechamente ligadas a las políticas orientadas al desarrollo económico y social. Tomar en cuenta a los organismos filantrópicos y no gubernamentales, puede ser útil en la formulación y la aplicación de políticas en ciencia y tecnología, sobre todo en contextos donde estamos observando nuevas dinámicas en el desarrollo tecnológico. 


\section{Referencias}

ABIR-AM, P. G. The Rockefeller Foundation and the rise of molecular biology. Nature Reviews - Molecular Cell Biology, vol. 3, pp. 65-70. 2002.

ARNOVE, R. Philanthropy and Cultural Imperialism. The Foundations at Home and Abroad. Indiana University Press. 1980.

ARNOVE, R. and PINEDE, N. Revisiting the "Big Three" Foundations. Critical Sociology, vol. 33, issue 3, pp.389-425. 2007.

AVENDAÑO, G. Entretien personnel effectué le 9 septembre 2009.

BOGDANCHIKOVA, N., AMAYA-CORONA, G., PINEDA-MARTÍNEZ, E., CATALAN, J., ARUELA, A., BAPTISTA, R., SALINAS-RAMÍREZ, A., HIRATA, G., ESTRADA-RIVERA, R. Desarrollo de calzado innovador de modelo Nanoplata para pie diabético y personas de actividades forzadas y durante largo tiempo. Conferencia NanoMex'09, 11 y 12 de noviembre, Ensenada, México. 2009.

BROWN, Jr.G.E. and SAREWITZ, D.R. Fiscal Alchemy: Transforming Debt into Research. Issues in Science and technology, vol.7, Fall, pp. 7076. 1991.

BROWN, E. R. Rockfeller Medicine in China: Professionalism and Imperialism, in: Arnove, R. (ed.). Philanthropy and Cultural Imperialism. The Foundations at Home and Abroad. Indiana University Press, pp. 123-146. 1980.

BUXRON. John Marshall And The Humanities In Europe: Shifting Patterns Of Rockefeller Foundation Support. Minerva, vol. 41, issue 2, pp.133-153. 2003.

CASAS, R. y DETTMER, J.A. Construyendo un paradigma de política científico tecnológica para México, in : Calva, J. L. (coord.), Educación, ciencia, tecnología y competitividad. Agenda para el desarrollo, vol. 10, Edit. Porrua y UNAM, México. 2007.

CUETO, M. Visions of Science and Development. The Rockefeller Foundation's Latin American Surveys of the 1920s, in: Cueto, M. (ed.), Missionaries of Science. The Rockefeller Foundation \& Latin America. Indiana University Press, pp. 1-22.1994. 
CUETO, M. Science under Adversity: Latin American Medical Research and American Private Philanthropy, 1920-1960. Minerva, vol. 35, issue 3, pp. 233-245. 1997.

ETZKOWITZ, $\mathrm{H}$. and LEYDESDORFF L. The dynamics of innovation : from National Systems and "Mode 2 » to a Triple Helix of university-industrygovernment relations. Research Policy, Vol. 29, issue 2, pp. 109-123. 2000.

FARIA, L. y da COSTA, M. C. Cooperação Científica Internacional: Estilos de Atuação da Fundação Rockefeller e da Fundação Ford. DADOS Revista de Ciências Sociais, Vol. 49, no. 1, pp. 159-191. 2006.

FITZGERALD, D. Exporting American Agriculture. The Rockefeller Foundation in Mexico, 1943-1953, in: Cueto, M. (ed.), Missionaries od Science. The Rockefeller Foundation \& Latin America. Indiana University Press, pp. 72-96. 1994.

FLORES-HERRERA, L.A. "Manufactura y análisis numérico de Sistemas Micro Electro Mecánicos". Tesis de doctorado en ciencias, ESIME-IPN, México. 2007.

FREIBURGHOUSE, A. Military. Forbes, vol. 167, issue 8, pp. 52-52. 2001.

FUMEC. Reporte de actividades 1992-1997. [Disponible en: http://www.fumec.org.mx]. 1998.

FUMEC. Reporte de actividades 1998-1999. [Disponible en: http://www.fumec.org.mx]. 2000.

FUMEC. Reporte de actividades 2000-2001. [Disponible en: http://www.fumec.org.mx]. 2000.

FUMEC. Reporte de actividades 2004-2005. [Disponible en: http://www.fumec.org.mx]. 2006.

FUMEC. Reporte de actividades 2006-2007. [Disponible en: http://www.fumec.org.mx]. 2008.

FUMEC. Reporte de actividades 2008-2009. [Disponible en: http://www.fumec.org.mx]. 2010.

GAD-EL-HAK, M. The MEMS Handbook. CRC Press. 2001.

GIBBONS, M., LIMOGES, C., NOWOTNY, H., SCHWARTZMAN, S., SCOTT, P. and TROW, M. The New Production of Knowledge: The Dynamics of Science and Research in Contemporary Societies. SAGE, London.1994. 
GONZÁlEZ-FERNÁNDEZ, S., y PÉREZ-ÍNIIGO, J.M. Los swaps deuda/naturaleza: estado del arte. Revista de Economía Mundial, vol. 18, pp. 231-243. 2008.

GUILHOT, N. Reforming the World: George Soros, Global Capitalism and the Philanthropic Management of the Social Sciences. Critical Sociology, vol. 33, issue 3, pp. 447-477. 2007.

HARWOOD, J. Peasant friendly plant breeding and the early years of the green revolution in Mexico. Agricultural History, vol. 83, issue 3, pp. 384410. 2009.

LOSEGO, P. et ARVANITIS, R. La science dans les pays non hégémoniques. Revue d'anthropologie des connaissances, Vol. $2, n^{\circ} 3$, pp. 334-342. 2008.

LUNDVALL, B.A. National Systems of Innovation. Towards a Theory of Innovation and Interactive Learning. Pinter Publishers, London. 1992.

MERTON, R.K. Social Theory and Social Structure. The Free Press [4a edición en español: Teoría y estructuras sociales, Ed. Fondo de Cultura Económica, México]. 1949 [2003].

PARMAR, I. American foundations and the development of international knowledge networks. Global Networks, vol. 2, issue 1, pp 13-30. $2002 a$.

PARMAR, I. To Relate Knowledge and Action: the Impact of the Rockefeller Foundation on Foreing Policy Thinking During America's Rise Globalism 1939-1945. Minerva, vol. 40, no. 3, pp. 235-263. $2002 b$.

PÉREZ, C. Revoluciones tecnológicas y capital financiero. La dinámica de las grandes burbujas financieras y las épocas de bonanza. México, Edit. Siglo XXI. 2004.

ROBLES-BELMONT, E. Las redes científicas como respuesta a la emergencia de las nanociencias y nanotecnologías. REDES - Revista de estudios sociales de la ciencia, Vol. 15, $\mathrm{n}^{\circ} 29$, Buenos Aires, Mayo de 2009, pp. 93-111. 2009.

ROBLES-BELMONT, E. «Les Fondations, acteurs de l'émergence des nouvelles technologies dans les pays non hégémoniques : le cas des micro et nanotechnologies au Mexique ». Tesis de doctorado en sociología industrial, Universidad de Grenoble, Francia. 2011.

SABATO, J.A., et Botana, N. La science, la technique et l'avenir de l'Amérique latine: analyse et stratégie. Tiers-Monde, Vol. 12, No. 47, pp. 579-594. 1971. 
SHRUM, W. Science and Story in Development: The Emergence of NonGovernmental Organizations in Agricultural Research. Social Studies of Science, Vol. 30, Issue 1, pp. 95-124. 2000.

SOLORZANO, A. The Rockefeller Foundation in Revolutionary Mexico: Yellow Fever in Yucatan and Veracruz, in: Coeto, M. (ed.), Missionaries of Science. The Rockefeller Foundation \& Latin America. Indiana University Press, pp. 52-71. 1994.

STAPLETON, D. H. Joseph Willits And The Rockefeller's European Programme In The Social Sciences. Minerva, vol. 41, issue 2, pp. 101-114. 2003.

TOENNIESSEN, G., ADESINA, A. and DeVRIES, J. Building an Alliance for a Green Revolution in Africa. Annals of the New York Academy of Sciences, vol. 1136, pp.233-242. 2008.

VESSURI, H. Foreign Scientists, the Rockefeller Foundation and the Origins of Agricultural Science in Venezuela. Minerva, vol. 32, issue 3, pp. 267-296. 1994.

VINCK, D. y ROBLES-BELMONT, E. Convergence dans les nanosciences et les nanotechnologies. Le cas des micro $y$ nanosystèmes. In B. Miège et $D$. Vinck. Les masques de la convergence. Enquêtes sur sciences, industries et aménagements. Archives des Editions Contemporaines, pp. 43-65. 2011. 